ARTÍCULOS ORIGINALES Rev Chil Salud Pública 2015; Vol 19 (1): 47-52
Sergio Bermedo

Departamento de Salud Municipalidad de Río Negro Río Negro, Chile serbemun@yahoo.es

\section{DIMENSIONES Y SIGNIFICADOS QUE ADQUIERE EL PROCESO SALUD/ENFERMEDAD/ATENCIÓN EN USUARIOS MAPUCHE - WILLICHE CON DIABETES MELLITUS E HIPERTENSIÓN ARTERIAL}

\author{
Dimensions AND MEANING SURROUNDING THE HEALTH-DISEASE- \\ CARE process for Mapuche-Williche patients With Diabetes \\ AND HYPERTENSION
}

\section{RESUMEN}

Las enfermedades cardiovasculares representan la primera causa de muerte en población adulta en Chile. En el caso de la población indígena, los resultados de estudios epidemiológicos comparativos muestran un peso relativo mayor de estas enfermedades en la población indígena que en la no indígena.

Objetivo: Describir las concepciones y prácticas terapéuticas tradicionales que convergen en los procesos de sanación de usuarios mapuche-williche diabéticos e hipertensos que consultan en los establecimientos de salud, ubicados en la jurisdicción del cacicado de Riachuelo, Rio Negro.

Material y Método: Estudio exploratorio-descriptivo de carácter socioantropológico que se inscribe en el paradigma interpretativista que articula el proceso salud/enfermedad/atención que desarrollan los pueblos originarios y las lógicas positivistas de la biomedicina. El muestreo fue no probabilístico. Se realizó estudio de caso y entrevista en profundidad. Para el análisis de los datos se utilizó el análisis categorial aplicado a las entrevistas, construyendo interpretaciones desde los sentidos y significados que los propios actores transparentan en sus discursos y prácticas culturales.

Resultados: El sistema cultural de salud mapuche-williche es relevado por los usuarios en un itinerario terapéutico que los lleva a complementar, alternar o sustituir la medicina occidental por la medicina mapuche para el tratamiento de la diabetes mellitus e bipertensión arterial.

Conclusiones: Las enfermedades cardiovasculares conceptualizadas por el modelo médico oficial-hegemónico no son parte de la matriz cultural mapuche-williche, constatándose dificultades en la adberencia al tratamiento impuesto por la biomedicina en usuarios que viven en territorios con alta concentración de población indígena.

Palabras clave: sistema médico mapuche-williche, enfermedades cardiovasculares, itinerario terapéutico, competencias interculturales.

Recibido 14 de octubre 2014; aceptado 12 de enero 2015

Financiamiento: Programa Salud y Pueblos Indígenas. Ministerio de Salud. Convenio Servicio Salud Osorno - Municipalidad de Río Negro. 


\section{ABSTRACT}

Cardiovascular diseases are the leading cause of death in adults in Chile. Comparative epidemiological studies show a relatively greater burden of these diseases amongst the indigenous population compared to the non-indigenous population.

Objective: To describe the concepts and traditional healing practices that converge in the healing process of mapuche-williche diabetic and hypertensive patients, treated in bealth facilities located in the jurisdiction of cacicado of Riachuelo, Rio Negro.

Material and Methods: exploratory-descriptive study, socio-anthropological in character, part of the interpretive paradigm that articulates the health/disease/care developed by indigenous peoples, and logical positivist biomedicine. Sampling was not probabilistic. Case study and indepth interviews were conducted. An examination of the interview categories was performed to construct interpretations from the sense and meaning that the subjects showed through their discourse and cultural practices.

Results: The mapuche-williche cultural health system is revealed by patients: we see a therapeutic journey that leads them to complement, switch or replace Western medicine with mapuche medicine for the treatment of diabetes mellitus and hypertension.

Conclusions: Cardiovascular diseases conceptualized by the hegemonic medical model are not part of the cultural mapuche-williche matrix, confirming the difficulties in following biomedical treatment for patients coming from areas with a dense indigenous population.

Keywords: mapuche-williche medical system, cardiovascular disease, diabetes, therapeutic itinerary, intercultural competence.

\section{INTRODUCCIÓN}

En las dos últimas décadas han surgido importantes iniciativas de investigación y de conocimiento con el objeto de comprender más cabalmente las condiciones epidemiológicas de los pueblos originarios del país, demostrándose que los usuarios de los pueblos indígenas tienen un perfil de morbimortalidad distinto al de la población no indígena. El Perfil Epidemiológico Básico de la Población Mapuche Residente en el Área de Cobertura del Servicio de Salud Osorno, entre sus hallazgos señala que: "La población mapuche de la Provincia de Osorno no solo presenta una tasa general de mortalidad más alta que la población no mapuche -al igual que en los demás territorios estudiados a través del proyecto de Epidemiología Sociocultural- sino que, además, ésta constituye la mayor tasa registrada hasta ahora para esta población". Situación que podría estar vinculada con "la pérdida de los factores protectores propios de la cultura mapuche y a la incorporación en situación desventajosa de la modernidad". En el caso de las enfermedades del sistema circulatorio la población mapuche de la provincia de Osorno tiene un riesgo de un $60 \%$ mayor de morir por este grupo de causa que los no mapuche. ${ }^{1}$

El Informe de la ONU sobre la Situación de los Pueblos Indígenas del Mundo, reporta sobre los "niveles alarmantes de diabetes. En todo el mundo más del $50 \%$ de los adultos indígenas de más de 35 años de edad padece diabetes tipo 2, y se prevé que estas cifras aumenten. En algunas comunidades indígenas, la diabetes ha alcanzado proporciones epidémicas y pone en peligro la existencia misma de la comunidad". ${ }^{2}$ Por su parte, la Federación Internacional de Diabetes señala que si bien esta patología está presente en todo el mundo, los pueblos indígenas soportan una carga desproporcionadamente mayor en este tipo de padecimientos. ${ }^{3}$

Para indagar acerca de la percepción que poseen los usuarios indígenas diabéticos e 
hipertensos sobre este tipo de enfermedades, y describir el conocimiento y las prácticas de la medicina ancestral que se integran a los proceso de salud convencional durante el itinerario terapéutico seguido por los usuarios, se realizó el estudio exploratorio - descriptivo con usuarios mapuche-williche que se mantienen en control en el Programa de Salud Cardiovascular (PSCV) en el Centro Comunitario de Salud Familiar (CECOSF) de Riachuelo y las Estaciones Médico Rurales (E.M.R.) de Millantue y Costa Río Blanco ubicados en el territorio cacical de Riachuelo.

\section{MATERIAL Y MÉTODO}

El universo son 320 personas, que corresponde al total de usuarios diabéticos e hipertensos en control en el PSCV, según el REM de junio del 2012.(i) Los criterios de inclusión son: ser hombre o mujer, diabético y/o hipertenso independiente del estado de compensación; con diagnóstico y tratamiento ingresados al Sistema de Información para la Gestión de Garantías de Salud (SIGGES), de acuerdo a la Ley 19.966 sobre el Régimen de Garantías en Salud. Tener al menos un apellido mapuche y/o estar su calidad de indígena acreditada ante la CONADI.

El diseño es no probabilístico de acuerdo el carácter cualitativo de la investigación. Las técnicas de recolección de la información fueron un cuestionario estructurado y una entrevista en profundidad semiestructura$\mathrm{da}$, siendo: "La entrevista uno de los medios para acceder al conocimiento, las creencias, los rituales de vida de una sociedad o cultura, obteniendo datos en el propio lenguaje de los sujetos". Ambos instrumentos tuvieron como ejes de interés: i) los conocimientos y prácticas de la medicina mapuche-williche, ii) los mecanismos de transmisión del conocimiento y prácticas de la medicina tradicional, iii) el itinerario terapéutico seguido por los usuarios mapuche-williche diabéticos e hi-

(i). REM. Registro Estadístico Mensual. Serie P-4. Junio 2012. pertensos, iv) la percepción de las enfermedades cardiovasculares y la relación establecida con el sistema de salud oficial. Para el análisis de la información, se utilizó el análisis categorial aplicado a las entrevistas en profundidad, construyendo dimensiones e integrando categorías y sus propiedades e indagando en las analogías y diferencias de los contenidos, teniendo presente que el estudio es de carácter exploratorio, cuyos hallazgos requieren una mayor profundización.

\section{RESULTADOS}

Del total de usuarios bajo control un 32,8\% es de origen mapuche-williche, los que registran una edad promedio de 67 años, incrementándose significativamente con la edad el número de personas indígenas diabéticas e hipertensas, concentrándose en un $45 \%$ en el grupo etario de 70 y más años, observándose un número mayor de mujeres respecto a los hombres.

Un $74 \%$ de los hogares tiene como ingreso principal pensiones bajo el régimen de cotizaciones previsionales y básicas solidarias, cuyo monto promedio es de 85 mil pesos mensuales y más del $65 \%$ se sitúa entre el primer y segundo quintil de ingresos, según los tramos de puntaje de la Ficha de Protección Social que define los quintiles de vulnerabilidad social.

De los usuarios encuestados un 13,4\% vive con su esposo(a) o hijos y el $83,6 \%$ lo hace con otros familiares, principalmente nietos o sobrinos. Un $76 \%$ no cursó la educación básica.

Del 32,8\% de los usuarios mapuche que se mantienen en control en el PSCV, un $64,9 \%$ ha consultado algún especialista de la medicina mapuche-williche y un $67 \%$ declara usar hierbas medicinales para tratar diversas dolencias; estableciéndose diferencias entre quienes tienen conocimientos generales de la medicina tradicional como parte de la construcción del conocimiento del cuidado familiar, y quienes poseen el kimün (sabiduría) en el tratamiento de enfermedades más complejas y ejercen en la comunidad el rol de machi, lawentuchefe, ngütamchefe, u otro afín. 


\section{DISCUSIÓN}

La enfermedad es universal como experiencia humana y particular en cada cultura en cuanto al desarrollo de modelos, procedimientos y el aprendizaje de especialistas para hacer frente a los padecimientos y recuperar el estado de salud.

Las categorías de enfermedad en la medicina mapuche-williche se relacionan con la transgresión de las leyes o norma del equilibrio y armonía establecida por el ad mapu (ley de la tierra) y el ad che (ley de la gente) o por el abandono de prácticas espirituales y culturales propias del pueblo mapuche-williche. "Las medicinas indígenas persiguen, en especial, la recuperación del 'ser humano' en su contexto cultural y no tanto del órgano dañado como hace la biomedicina". ${ }^{5}$

Es parte del discurso de los usuarios señalar que la diabetes y la hipertensión son "enfermedades que no se conocían antes", que llegaron con la incorporación de alimentos procesados con alta cantidad de grasa y azúcar propios de la vida moderna. De acuerdo al origen percibido por los usuarios, éstas no son enfermedades propias de los mapuchewilliche y corresponderían a "enfermedades introducidas" por el contacto interétnico, que Luca Citarella clasifica como "wingka kutran" y que tienen un tratamiento basado principalmente en elementos químicos "invasivos” para el organismo y provocan efectos adversos: Mucha pastilla, se daña el estómago. Imagínese tomando pastilla todos los días, esa pastilla va quedando abi. ¿Cuántos dias pueden pasar para que se disuelva el omeprazol que viene con ese plástico? (E4MUR).

Otra causa identificada por los usuarios es el desapego del hombre (che) con la naturaleza y el abandono de los hábitos tradicionales de alimentación. Una de las personas refiere que la modificación de sus patrones alimentarios tradicionales ocurrió cuando: mi mamá ya no bizo más catuto; cuando ella dejó de hacer, porque ya no lo podia hacer y nosotros ya no lo haciamos, porque después ya empezó a escasear el trigo. Habia que estarlo comprando, porque antes mi abuelito lo cosechaba. Antes se sembraba y se cosechaba el trigo y abi se hacia eso. Y también las papas, se hacía milcao, comíamos mucho el trutruyeco, que le dicen. Eso que se hace en palo, se hace como un rollito y se comía con miel, con dulce (E10MUCRB).

Investigaciones de prevalencia e incidencia de la diabetes en comunidades indígenas han concluido que "algunas poblaciones que todavía viven con un estilo de vida muy tradicional tienen una prevalencia relativamente baja" en este tipo de enfermedades. ${ }^{7}$

Desde la primera consulta en los establecimientos de salud los usuarios siguen un "itinerario terapéutico", el que según Nicole Alice Sindzingre considera "todos los procesos que se llevan a cabo para buscar una terapia, desde que aparece el problema, se ponen en marcha diversos tipos de interpretación y cura, y se utilizan diversas instancias terapéuticas, institucionales o no, todo ello en un contexto de pluralismo médico". ${ }^{8}$

El transitar del usuario mapuche-williche de un sistema a otro, considera:

a. Consultar al profesional alópata de la salud oficial para el diagnóstico y tratamiento.

b. El usar medicina mapuche-williche con o sin consultar a sus especialistas: varias veces que me hice los exámenes, me resultó. $Y$ las yerbas que sigo tomando; tomo la limpia plata, que es para purificar la sangre, la pata de caballo, el natre igual lo consumo en tecito, porque eso igual me baja todo eso: la glicemia. [...] me he tomado la glicemia y he tomado eso y sí me ha bajado la glicemia. He estado tomando, supongamos, una semana antes de tomarme la glicemia y he llegado con glicemias bajas (E1MUR).

c. Teniendo experiencia con ambos sistemas de atención los usuarios deciden, con independencia de las indicaciones médicas de la primera consulta, estrategias de complementariedad, sustitución y/o alternancia de la terapia biomédica por la medicina mapuche-williche, con el objetivo de apoyar el tratamiento farmacológico, evitar efectos adversos que provocan los fármacos o probar la efectividad de las hierbas 
medicinales: a mí siempre me gusta así, porque acompañe a los remedios que le dan en la Posta, ayuda harto [...] si no siento nada de la cuestión de la presión, muy bueno para la presión, lo tomo con las pastillas... (E9HUR)

En la relación médico-paciente que se establece en la salud oficial, el primero tiende a desconocer el contexto cultural de los usuarios y el uso que realizan de la medicina tradicional y solo exige el uso de la biomedicina: los médicos no me dicen nada por tomar hierbas. Lo unico que me obligan que tome todos estos remedios (E8MUR). Las personas refieren que las indicaciones recibidas no pueden ser contradichas, pues el médico siempre tendrá la razón, [...] si uno no le gana a ellos (E5MUCRB). De las opiniones de los usuarios se desprende un discurso impositivo y reduccionista de la salud que busca perpetuar el modelo biomédico, donde los médicos se apoderan del lenguaje: la persona enferma queda privada de palabras significativas para expresar su angustia, que aumenta más aún con la mitificación lingüística. ${ }^{9}$ Se aprecia un cumplimiento de tratamiento centrado en la propuesta del médico más que una adherencia terapéutica producto de acuerdo dinámico entre el profesional y el usuario.

No obstante el Ministerio de Salud declare el "respeto y la consideración de la cosmovisión de los pueblos, sus modelos de salud y sus itinerarios terapéuticos [...]", ${ }^{10}$ el plan de control y tratamiento de las enfermedades crónicas obliga a los usuarios a que asistan regularmente a retirar sus remedios, pero una vez en su casa las personas determinan autónomamente el reducir, aumentar o espaciar las dosificaciones o lisa y llanamente abandonan el tratamiento: cuando eché de ver que me hacian mal, ya no las tomé; los dejé. Una semana sin tomarla y ya eché de ver que cambió mi cuerpo. O sea, en la noche no dormía casi [...] Notaba que me hacian mal, cuando lo tomaba. Por ejemplo, lo tomaba en la mañana, como ser el paracetamol una al día, la aspirina una al día, las otras pastillas también. Todas una al dia, asi que todas esas pastillas las juntaba y las tomaba de un viaje (E10MUCRB).

\section{CONCLUSIONES}

Concebido el sistema cultural de salud mapuche-williche como una construcción cultural y simbólica para dar respuesta a las experiencias de enfermedad, dolor, sufrimiento y muerte, desde la cosmovisión mapuche, la denominación de diabetes mellitus y/o hipertensión arterial -empleadas por la salud convencional- no son parte de la matriz cultural mapuche-williche. Al no haber identidad cultural con este tipo de enfermedades y conocidos los efectos adversos que provoca la medicación, los usuarios optan por no dar continuidad a tratamientos prolongados tomando distancia del modelo explicativo de la biomedicina y de su cartera de servicios, poniéndose en duda la pertinencia cultural del modelo médico hegemónico, recuperando vigencia los conocimientos y prácticas tradicionales de atención de salud. ${ }^{11}$

Este modelo hegemónico no reconoce las prácticas médicas tradicionales ni tampoco le interesa la resignificación que le dan los usuarios al tratamiento farmacológico para la diabetes y la hipertensión: según dicen que las pastillas hacen más mal que las agua, [...] al final de tanto tomar esas pastillas se van acumulando en el estómago y por eso más dicen que proviene el cáncer (E10MUCRB). Las barreras "en el lenguaje, en la falta de reconocimiento sanitario de las familias y las diferentes expectativas de la relación médicopaciente" han sido hallazgos frecuentes en estudios con población mapuche que permiten concluir que las brechas entre los equipos de salud y los usuarios tienen su origen en los modelos etiológicos culturales de salud y enfermedad y a la pertenencia a estratos socioeducacionales diferenciados. Esto denota la ausencia de competencias culturales de los servicios de salud para trabajar en contexto pluriculturales. ${ }^{12}$

El Convenio 169 ratificado por Chile el año 2009 establece que el Estado debe "velar por que se pongan a disposición de los pueblos interesados servicios de salud adecuados [...], a fin de que puedan gozar del máximo nivel posible de salud física y mental" ${ }^{13}$ siendo imperioso avanzar en la 
transversalización del Enfoque Intercultural en los Programas de Salud, asumiendo los equipos de salud que el "modelo científico occidental no es el único deseable y válido", ${ }^{(14)}$ que existe un complejo mundo de creencias y valores, normas, conocimientos y comportamientos ligados a la experiencia de salud y enfermedad que involucra: a) la construcción cultural del padecimiento (illness), b) la enfermedad como una condición biológica (disease) y c) el contexto social donde se vinculan la experiencia personal del usuario con su dolencia y el ejercicio de la medicina (sickness), dimensión biológica, cultural y social de la enfermedad. ${ }^{15} \mathrm{El}$ proceso salud/enfermedad no puede ser entendido al margen del contexto cultural de la persona; ambas racionalidades se deben articular: la científica dicotómica-binaria que todo lo objetiva y la mapuche-williche holística centrada en la persona y en el buen vivir (küme - mongen).

La salud intercultural como estrategia de salud pública en territorio mapuche-williche implica necesariamente un cambio en el paradigma del ejercicio de la medicina oficial, identificando los factores protectores y recursos terapéuticos culturales y reconociendo el corpus ideológico que forma parte indisoluble del sistema médico mapuche-williche. ${ }^{16}$ Esto conlleva una "modificación de los programas de salud [...] para darles coherencia con la cosmovisión y prácticas de salud propias de los pueblos indígenas" ${ }^{17}$ y una aproximación al universo cultural de los usuarios desde el enfoque de la epidemiología sociocultural y del derecho a la salud de los pueblos indígenas.

\section{REFERENCIAS}

1. Pedrero M. Perfil epidemiológico básico de la población mapuche residente en el área de cobertura del Servicio de Salud Osorno. Serie Análisis Situación de los Pueblos Indígenas de Chile. Ministerio de Salud. Santiago, Chile. 2012, 64 pp.
2. ONU. Informe Mundial de la Situación de Salud de los Pueblos Indígenas. Foro Permanente de las Naciones Unidas para las Cuestiones Indígenas. Nueva York. 2010. Capítulo V Salud, 164 pp.

3. 7. Federación Internacional de Diabetes, Atlas de la Diabetes. Bélgica. 2013, $6^{a}$ ed. 80 pp.

4. Rodríguez G, Gil J, García E. Metodología de la Investigación Cualitativa. España, 1996, pp. 167-168.

5. Fernández G. Salud e interculturalidad: Sugerencias para organizaciones de salud en contextos indígenas, a partir de una experiencia boliviana. $R D T P, \mathrm{LX}, 2,2005$, pp. 29-53.

6. Citarella L. et al. (Comp.). Medicinas y culturas en la Araucanía. Santiago de Chile: Trafkin - Cooperación Italiana - Editorial Sudamericana, 2000, pp. 129-140.

8. Perdiguero E. Una reflexión sobre el Pluralismo Médico. Salud e Interculturalidad en América Latina. Antropología de la salud y Crítica Intercultural. Ecuador: Ediciones Abya-Yala, 2006, 41 pp.

9. Illich I. Némesis Médica. Barcelona, 1975, $146 \mathrm{pp}$.

10. Ministerio de Salud. Orientaciones para la Implementación del Modelo de Atención Integral de Salud Familiar. 2012

11. Menéndez E. La Enfermedad y la Curación. ¿Qué es Medicina Tradicional? Revista Alteridades, 1994, pp. 71-83.

12. Alarcón-Muñoz AM, Vidal-Herrera AC. Dimensiones culturales en el proceso de atención primaria infantil: perspectivas de las madres. Salud Pública Mex 2005; 47: 440446.

13. Ministerio de Salud. Convenio 169 Sobre Pueblos Indígenas y Tribales de la OIT. 2009. Art. 25.

14. 17. Ministerio de Salud. Orientaciones para la Programación en Red. 2013.

15. Ibacache J, Leighton A, Torres M. Síndromes Culturales en el Archipiélago de Chiloé. 2010. 15 pp.

16. ONU. Declaración de las Naciones Unidas sobre los Derechos de los Pueblos Indígenas. 2007. Art. 24. 